\title{
ANALYSIS OF FLAT PLATE VIBRATIONS \\ BY VARYING FRONTAL AREA TO THE FLOW
}

\author{
ShravanKoundinya Vutukuru, Janis Viba, Igors Tipans, Ilmars Viksne, Martins Irbe \\ Riga Technical University, Latvia \\ vshravankoundinya1989@gmail.com, janis.viba@rtu.lv, igors.tipans@rtu.lv, \\ ilmars.viksne@rtu.lv, martins.irbe@rtu.lv
}

\begin{abstract}
Fluid rigid body interaction is commonly occurring phenomenon and this phenomenon is of high importance in many engineering applications. The main objective of the present paper is to analyse vibrations due to fluid -rigid body interactions by inclining the frontal area of flat plate to flow. As understood from the existing research, the main challenge is the understanding of non-stationary fluid body interactions. Interaction analysis, optimization and synthesis tasks include space - time programming methods and approximate analytical methods. This article discusses an approximate analytical method in which the object's interaction with fluid flow is divided into two parts in the fluid body interaction space. The first part is the frontal pressure side that arise as a result of change of momentum in the system that can be conveniently represented in a differential form. The second part includes the idea of describing the interaction behind the plate as a certain thin (vacuum) side was accepted. This thin vacuum side also depends on the frontal area flow interaction. The use and precision of the approximate analytical method was verified by experiments in the wind tunnel. The method was used for good analysing of varying frontal area (until flattening) of flat plate in fluid flow. The main parameters for motion excitation are the changes in plate-flow interaction area, velocity and angle of inclination of the flat square plate to the fluid flow. Experiments were performed at $10 \mathrm{~m} \cdot \mathrm{s}^{-1}$ keeping in view the wind speeds that were observed in the past in Riga, Latvia.
\end{abstract}

Keywords: energy generation, flow interaction, optimization.

\section{Introduction}

Fluid, non-stationary rigid body interaction is a challenging task; unfortunately, this interaction phenomenon is not extensively focussed. The subject of the study is the determination of fluid interactions with rigid body in non-stationary motion in a fluid flow. The only additional task is complicated by the fact that the flow rates of the body and fluid are vector in different directions. To simplify the calculations for analysis, optimization and synthesis of new structural systems, our present work offers a mathematical model approximated without considering the viscous effects of fluid. The interaction fluid space is divided into two: one on the pressure side and the other on the vacuum - thin side just behind the body as shown in Fig. 2.

At first numerical simulations were performed for presence of both interaction sides (pressure and vacuum) in fluid space in 3D. Computations are performed in ANSYS AIM 19.2 for flat sqaure plate of side $0.16 \mathrm{~m}$. The steady state RANS is solved using $\mathrm{k}-\varepsilon$ realizable turbulence model. A tetrahedron mesh with 178,855 elements (cells) for the flat plate was used Fig. 1. Residuals for Continuity and Momentum conservation in $x, y, z$, directions were monitored and all the residuals in all the cases converged to $10^{-4}$.

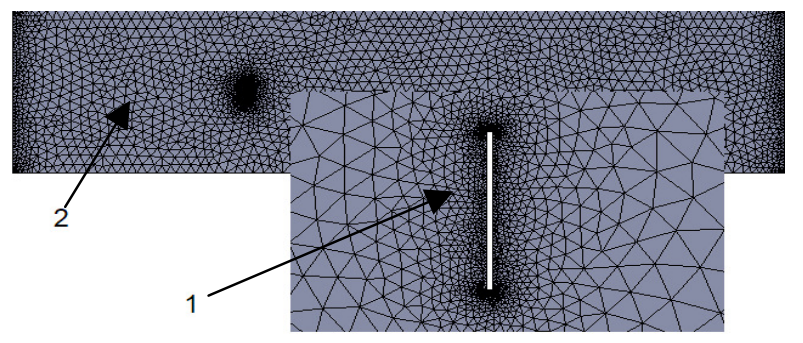

Fig. 1. Mesh Domain and close view of the mesh with the flat plate: 1 - plate; 2 - mesh domain

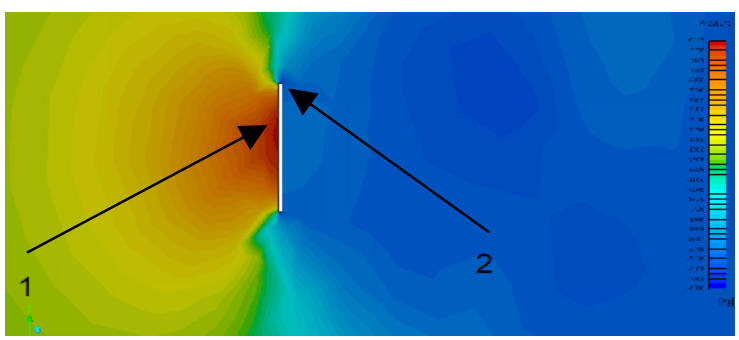

Fig. 2. Pressure distribution around the flat plate for positioning angle $0^{\circ}: 1$ - pressure side; 2 - Vacuum side

Computer modelling results for presure distribution around the flat plate for two different angles $\beta=0$ and $\pi / 4$ are shown in Fig. 2, Fig. 3. 
The following important conclusion can be drawn from the static pressure distribution for the flat plate is that the vacuum side: the thin side just downstream of the plate along the entire boundary region is approximately constant.

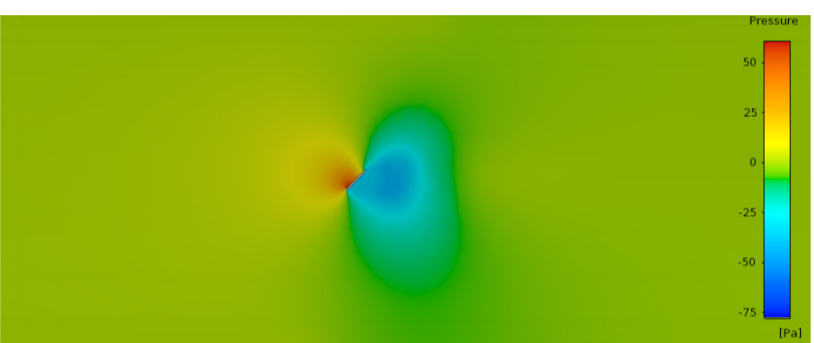




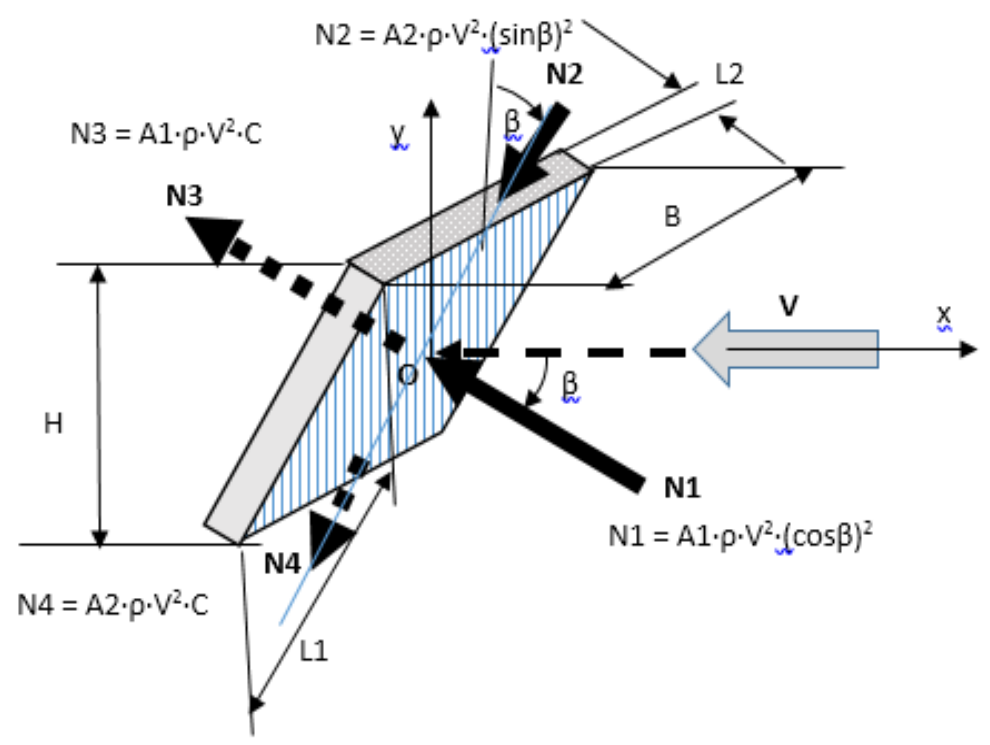

Fig. 7. Model in the case of rectangle flat plate: $L 1, B$ - length of flat plate sides; $L 2$ - thickness of plate; $A 1, A 2$ - area of sides; $N 1, N 2$ - centre forces of additional pressure in frontal sides;

$N 3, N 4$ - centre forces of vacuum (thin) side; $V$ - flow velocity

Vacuum (thin) side along the edge of the body is aproximately constant pressure. That is proportional to the density $\rho$ multiplied by the flow velocity square in the following forms (5), (6):

$$
\begin{aligned}
& N 3=A 1 \cdot v^{2} \cdot \rho \cdot C ; \\
& N 4=A 2 \cdot v^{2} \cdot \rho \cdot C,
\end{aligned}
$$

where $C$-constant, to be found out experimentally or by computer modelling.

At the centre of plate $O$ from equations (3) - (6) can be obtained two components of interaction forces (7), (8):

$$
\begin{gathered}
F x=-\mathrm{H} \cdot \mathrm{B} \cdot v^{2} \cdot \rho \cdot\left[C+\frac{\cos (\beta)^{3}+d \cdot \sin (\beta)^{3}}{\cos (\beta)+d \cdot \sin (\beta)}\right] \\
F y=\mathrm{L} 1 \cdot \mathrm{B} \cdot v^{2} \cdot \rho \cdot\left[C \cdot(\sin (\beta)-d \cdot \cos (\beta))+\sin (\beta) \cdot(\cos (\beta))^{2}+d \cdot(\cos (\beta))^{3}\right] .
\end{gathered}
$$

Here (Fig. 7.):

$$
H=L 1 \cdot(\cos (\beta)+d \cdot \sin (\beta)), d=\frac{L 2}{L 1},
$$

where $F x$ - in the direction of $x$ axis force component, named in fluid dynamics as Drag force; $F y$ - parallel to $y$ axis force component, named in fluid dynamics as Lifting force [5];

$H$ - section height perpendicular to flow.

From Equation (7), (8) coefficient $C$ is obtained as discussed below.

\section{Model verification by $2 \mathrm{D}$ simulation}

For coefficient C, 2D numerical simulations in SolidWorks flat plate drag interaction is investigated.

From the fluid Dynamics analysis (pressure graph), the interaction force is constantly fluctuating within certain limits, which depend on the angular $\beta$ values: at smaller angle, the amplitude of the fluctuations small and is greater at higher $\beta$ values. Therefore, only the mean values of interactions are analyzed here. Accordingly, part of interaction $D 1(\beta)$ as mentioned was found out by the step series (10): 


$$
\begin{aligned}
& D 1(\beta)=3.7266204256 \cdot(\beta)^{3}-1.5249241156366900458 \cdot(\beta)^{4}-0.1013532598492 \cdot(\beta)^{5}+ \\
& -2.81293523749905363 \cdot(\beta)^{2}+0.2823061 \cdot(\beta)+1.5
\end{aligned}
$$

For $(\beta=0)$, that coefficient in equation (7) is $C=0.5$, obtained from (10) and formula (7) can be used in form (11), (12):

$$
\begin{aligned}
& F x=-\mathrm{H} \cdot \mathrm{B} \cdot v^{2} \cdot \rho \cdot[D 2(\beta)] \\
& D 2(\beta)=\left[0.5+\frac{\cos (\beta)^{3}+d \cdot \sin (\beta)^{3}}{\cos (\beta)+d \cdot \sin (\beta)}\right]
\end{aligned}
$$

Accordingly, for (10), (11) Drag force coefficients $D F 1(\beta)$ and $D F 2(\beta)$ will be (13) (14):

$$
\begin{gathered}
D F 1(\beta)=D 1(\beta) \cdot(\cos (\beta)+d \cdot \sin (\beta)), \\
D F 2(\beta)=0.5 \cdot(\cos (\beta)+d \cdot \sin (\beta))+(\cos (\beta))^{3}+d \cdot(\sin (\beta))^{3} .
\end{gathered}
$$

Precision of approximation (7) with $C=0.5$ shown in Fig. 8 .

$$
\operatorname{Percent} 12(\beta)=\frac{|D F 2(\beta)|-|D F 1(\beta)|}{D F 1(0)} \cdot 100 .
$$

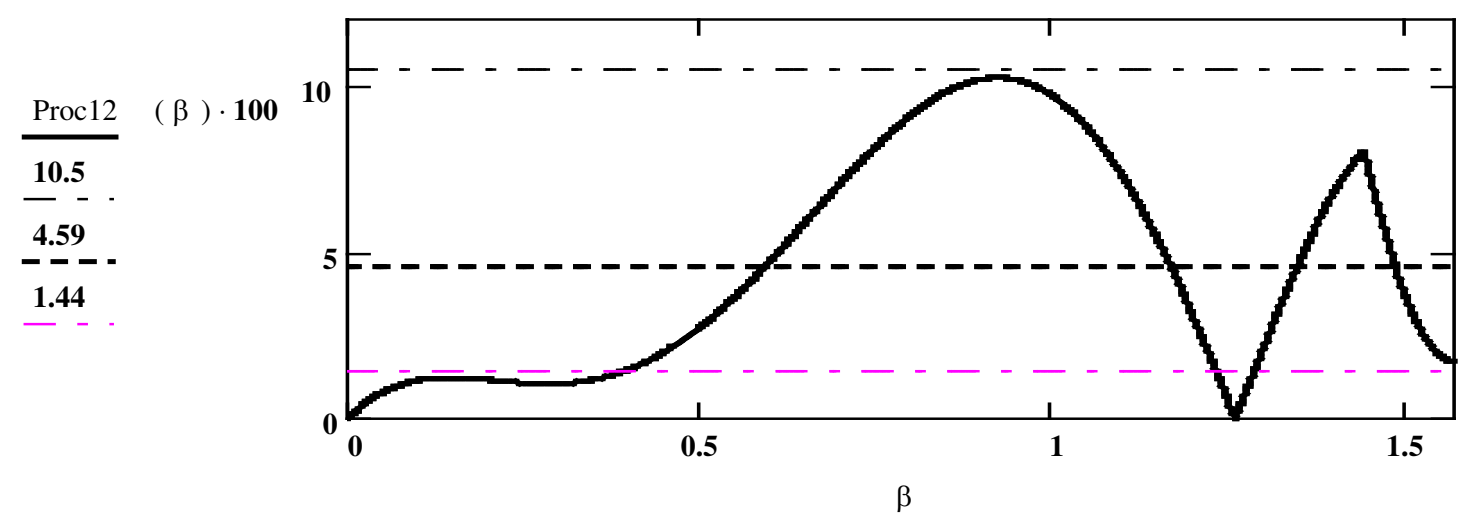

Fig. 8. Precision of rectangle flat plate model in per cents, percentage error against $\beta$ radians: 10.5 - maximal error \%; 4.59 -mean value of error $\% ; 1.44$ - mean value of error $\%$ in region $\beta=0-$ $\pi / 4$.

\section{Model verification by ANSYS followed by experiments}

For 3D square flat plate, the equation (7) is expressed as:

$$
F x=\frac{A \cdot V^{2} \cdot \rho}{2}\left(C+\frac{\cos (\beta)^{3}+d \cdot \sin (\beta)^{3}}{\cos (\beta)+d \cdot \sin (\beta)}\right),
$$

where $A$ - area of the body subjected to fluid flow,

From the computation results for drag force at different angles of $\beta$, when fitted to a curve, the equation (16) obtained is

$$
D 3(\beta)=-8.10^{-12} \cdot \beta^{5}+2.10^{-8} \cdot \beta^{4}+2.10^{-7} \cdot \beta^{3}-0.0004 \cdot \beta^{2}-0.0013 \cdot \beta+2,0463
$$

Similarly, for drag force experiments at $10 \mathrm{~m} / \mathrm{s}$, the results when approximated in the form of a curve, $5^{\text {th }}$ degree polynomial, resulted in equation (17) as shown in Fig. 9.

$$
D 3(\beta)=10^{-11} \cdot \beta^{5}+2.10^{-8} \cdot \beta^{4}+10^{-7} \cdot \beta^{3}-0.004 \cdot \beta^{2}-0.0016 \cdot \beta+2,1796 .
$$


From equation (15), calculating the interaction Force (Drag force) for the given geometry, the value of constant $C=0.31$ is obtained for $\beta=0^{0}$. Thereby, the equation for plate in 3D for motion analysis at $\beta=0^{\circ}$ is given by equation (18). The value 1.31 is taken for co-efficient of interaction. It was observed that the maximum error measured for Drag force at $\beta=0^{\circ}$, in computations and experiments was $\sim 6 \%$ as analysed from Fig. 9. Additional information from the simulations is given in Fig. 10, Fig. 11.

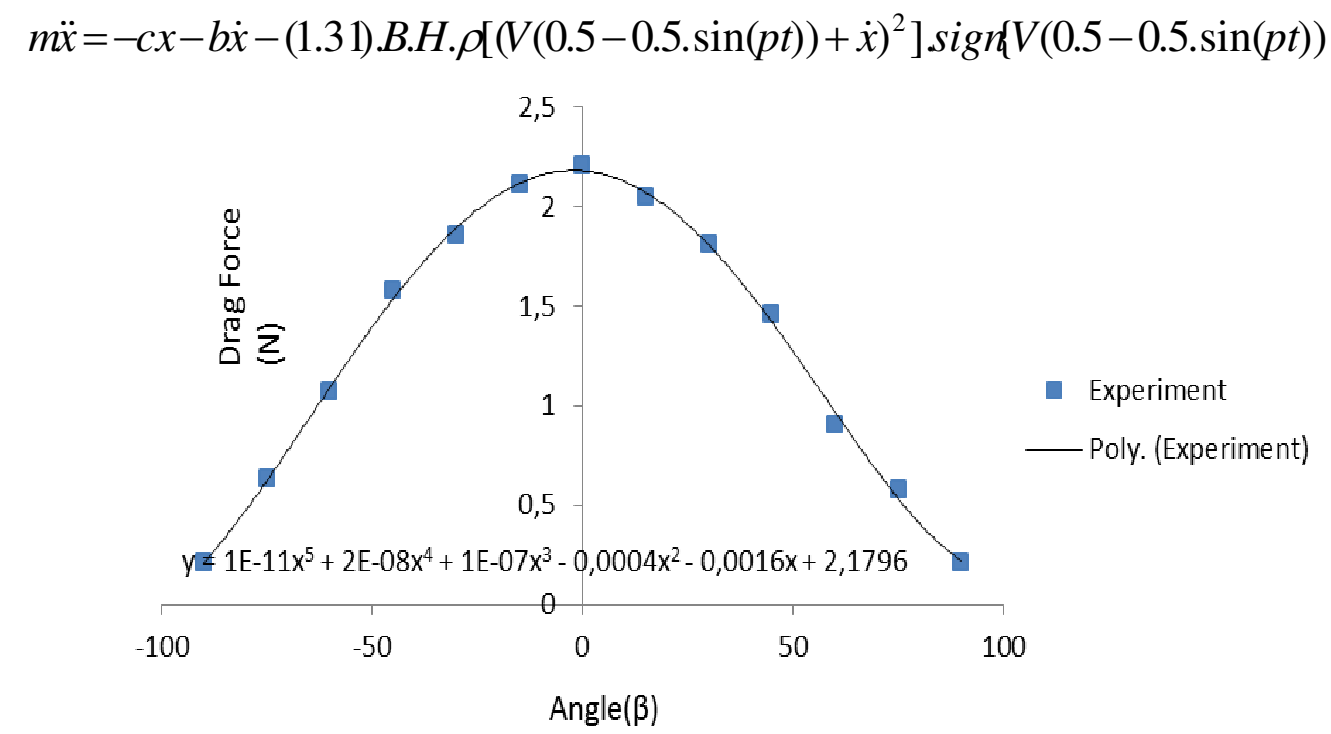

Fig. 9. Drag force experiment results for flat plate at velocity $10 \mathrm{~m} \cdot \mathrm{s}^{-1}, \beta$ degrees

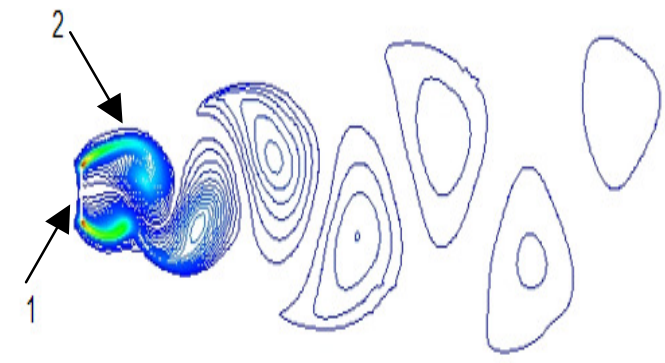

Fig. 10. Vorticity contour for flat plate at $\boldsymbol{\beta}=\mathbf{0}^{\mathbf{0}}: 1$ - flat plate; 2 - flow separation (vortex street formation)

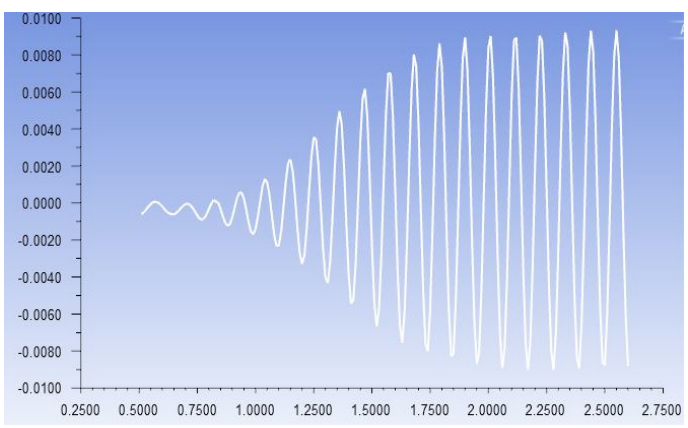

Fig. 11. Coefficient of Lift flow for $\left(\boldsymbol{\beta}=\mathbf{0}^{\circ}\right)$ as a function of time (varies within a large range around zero)

\section{Vibration analysis by Mathcad and Working Model 2D}

An example of a flat plate motion analysis in 2D space by Mathcad is shown in Fig. 12-14. The system considered consists of the mass of the plate, the spring, the energy generator and the flow, according to the equation (19),

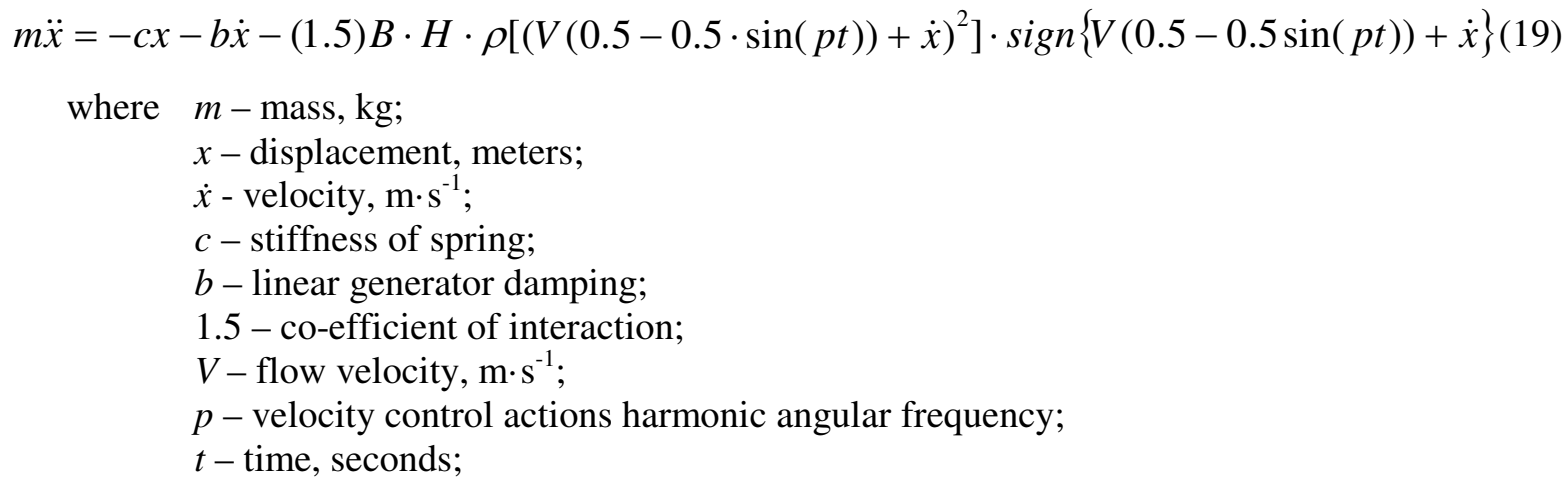


$B$ - depth of body, meters;

$H$ - length of body perpendicular to flow.

As shown here (19), flow control is selected as a function of harmonic velocity $V$ change. Similar modeling results with the Working Model according to equation (19) are shown in Fig. 15-17.

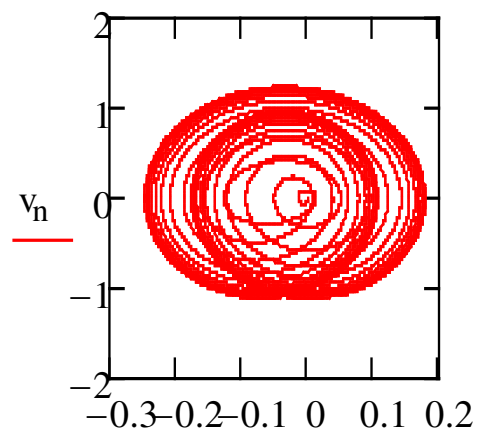

$\mathrm{x}_{\mathrm{h}}$

Fig. 12. Motion graphic in phase plane before resonance

Velocity $V_{n}\left(\mathrm{~m} \cdot \mathrm{s}^{-1}\right)$ against displacement, $x_{n}(\mathrm{~m})$

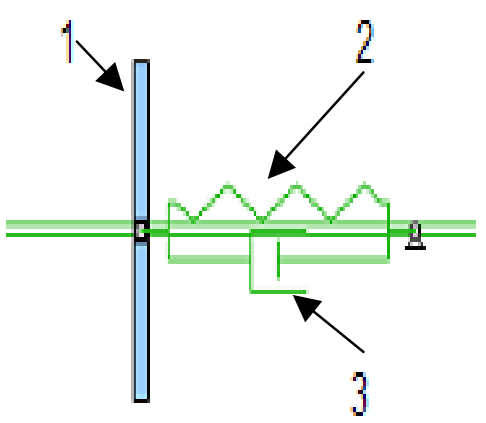

Fig. 15. Working Model 2D

elements: 1- flat plate;

2 - spring; 3 - generator's damper

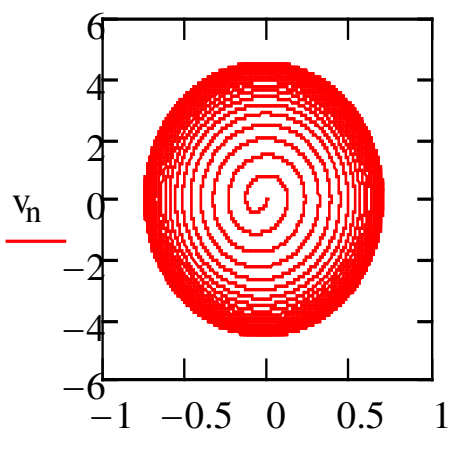

$x_{n}$

Fig. 13. Motion graphic in resonance. Velocity $V_{n}\left(\mathrm{~m} \cdot \mathrm{s}^{-1}\right)$ against displacement, $x_{n}(\mathrm{~m})$

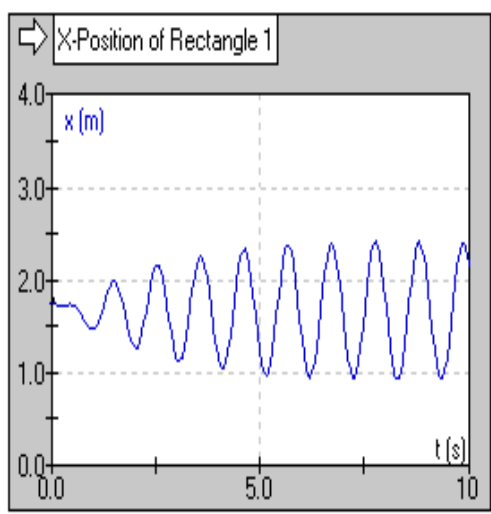

Fig. 16. Position of plate centre. Displacement $x(\mathrm{~m})$ against time, $t(\mathrm{~s})$

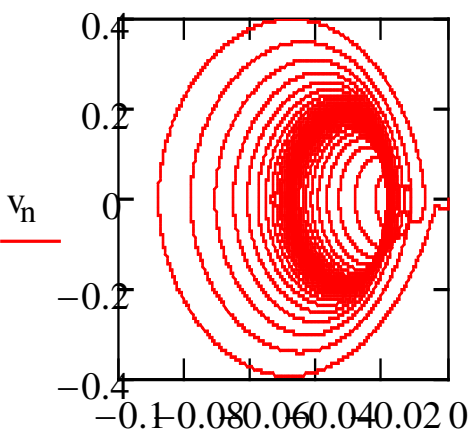

$x_{n}$

Fig. 14. Motion graphic after resonance. Velocity $V_{n}\left(\mathrm{~m} \cdot \mathrm{s}^{-1}\right)$ against displacement, $x_{n}(\mathrm{~m})$

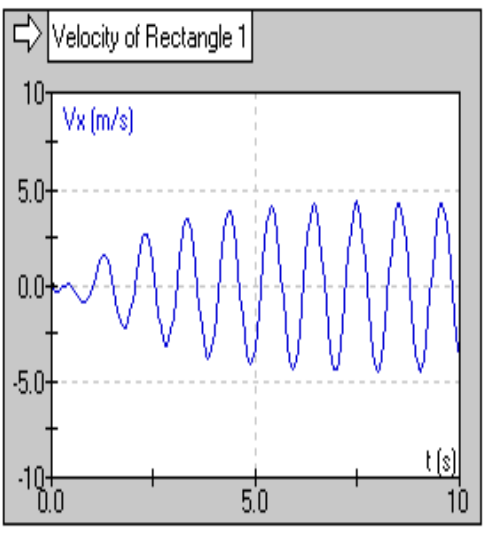

Fig. 17. Plate velocity in time.

Velocity $V_{\mathrm{x}}\left(\mathrm{m} \cdot \mathrm{s}^{-1}\right)$ against time, $t(\mathrm{~s})$

\section{Results and discussion}

1. In the present work a new method was developed for calculating approximate flow-flat plate interaction using the laws of classical mechanics.

2. The essence of the method is based on the evidence of numerical modelling that the interaction an be split separately on the sides of the pressure and then on the thin -vacuum behind the body.

3. The most interesting is of the interaction of the thin vacuum side just behind the plate which was almost constant, depends only on the flow rate.

4. It is important to note the fact that the proposed method is intended for analysis of non-stationary flow and object interaction and motion synthesis with the purpose of extracting energy from the fluid flow.

5. The flat plate analysis showed the known fact that in the case of stationary body the flow bifurcation begins on the collection side: pronounced pulsations appear perpendicular to the initial speed (Fig. 11). 


\section{Conclusions}

1. The method developed in the thesis allows performing tasks of analysis, optimization and synthesis in the interaction of objects with fluids in a simplified way.

2. This means there is no need to use time-space intensive computing programs.

3. The developed theory can be used in calculations of flying or diving robot systems, as well as in the extraction of energy from the fluid flow.

\section{References}

[1] Clancy L.J., Aerodynamics. London: Publishing by Pitman, 1975. 610p.

[2] Meriam J. L., Kraige L.G., Bolton J. N. Engineering Mechanics: Dynamics, 8th Edition, Wiley, 2015. $736 \mathrm{pp}$.

[3] Goldstein H., Poole C.P., Safko J.L. Classical Mechanics. Third Edition, Addison Wesley, $2000.625 \mathrm{p}$.

[4] Sears W.R. Introduction to Theoretical Aerodynamics and Hydrodynamics, American Institute of Aeronautics and Astronautics, Reston, VA, 2011.203p.

[5] Hoerner S. F. Fluid-Dynamic Drag.1965.

[6] Vība J., Beresņevičs V., Noskovs S., Irbe M. Investigations of Rotating Blade for Energy Extraction from Fluid Flow. Vibroenginesering PROCEDIA, vol. 8, 2016. pp. 312-315.

[7] Flat Plate Drag [online][16.02.2019] Available at: https://www.engineersedge.com/fluid_flow/rectangular_flat_plate_drag_14036.htm

[8] Drag force on a flat plate [online][16.02.2019] Available at: https://physics.stackexchange.com/questions/138166/drag-force-on-a-flat-plate. 